\title{
Über die Bestimmung des gelösten Sauerstoffs in verunreinigten Wässern.
}

Von

\author{
L. W. Winkler, Budapeŝt.
}

\author{
[Eingegangen am 19. November 1914.]
}

Der gelöste Sauerstoff läßt sich in einem reinen natürlichen Wasser mit der jodometrischen Methode sehr genau bestimmen. Enthält aber das Untersuchungswassser Nitrite und gleichzeitig in reichlicher Menge organische Stoffe, so zeigen sich gewisse Schwierigkeiten. Es wurden zwar, um diesen Mangel der Methode zu beheben, schon verschiedene Vorschläge ${ }^{1}$ ) gemacht, es könnte aber die hier zur Beschreibung gelangende neue Vorbehandlung ihrer leichten Ausführbarkeit halber, verbunden mit gehöriger Genauigkeit, für die Praxis dennoch von Wert sein.

Bei diesem zur Untersuchung von Nitrite und organische Stoffe enthaltendem Wasser bestimmten Verfahren werden die störenden Stoffe vorher unschädlich gemacht, dann die Sauerstoffbestimmung mit kleinen Änderungen in ihrer alten Form ausgeführt.

Wird zu einem verunreinigten Wasser etwas $\mathrm{Ch}$ lorkalk $\mathrm{r}_{\mathrm{s}} \mathrm{u} \mathrm{ng}$ und $\mathrm{Sch}$ w e felsäure hinzugefügt, so oxydiert die unterchlorige Säure bezw. das wirksame Chlor die salpetrige Säure fast sofort zu Salpetersäure; es werden bei dieser Behandlung, wenn auch langsamer, gleichzeitig auch die organischen Stoffe zu Verbindungen umgewandelt, die weiterhin kaum mehr Störungen verursachen. Es mula aber, bevor die Sauerstoffbestimmung ausgeführt wird, für das Entfernen des überschüssigen wirksamen Chlors gesorgt werden; als geeignet hierzu erwies sich Kalium rhodan id. Die Sauerstoffbestimmung wird nun in bekannter Weise vorgenommen, mit dem Unterschiede, daß statt der Manganchlorürlösung Manganosulfatlösung und zum Ansäuern statt Salzsäure Schwefelsäure genommen wird. Die Versuche ergaben nämlich, daßo, wenn man wie gewöhnlich Salzsäure zum Ansäuern benutzt, die Ergebnisse nicht ganz zufriedenstellend sind; wurde aber zum Ansäuern Schwefelsäure genommen, so hatte ein geringer Überschuß des Rhodanids fast keinen Einfluß auf die Ergebnisse. Aus demselben Grunde wurde die Manganchlorürlösung durch Manganosulfatlösung ersetzt. Es möge aber betont werden, daß im Untersuchungswasser ein Chlor-IonGehalt bis zu etwa $1000 \mathrm{mg}$ im Liter durchaus nicht störend wirkt.

Ob man nun das hier zur Beschreibung gelangende Verfahren in Gebrauch nebmen wird oder nicht, so wird man auch beim Verbleiben bei dem bisherigen Verfahren die Salzsäure doch gerne mit etwa 50\%-iger Schwefelsäure vertauschen. Es entfallen nämlich beim Gebrauch der geruchlosen verdünnten Schwefelsäure die Unannehmlichkeiten, durch welche der Analytiker beim Arbeiten mit rauchender Salzsäure belästigt wird. Auch beim Untersuchen nitrithaltiger Wässer nach der bisherigen Methode, also bei Verwendung von kaliumjodidfreier Natronlauge, kann zum Ansäuern Schwefelsäure genommen werden, in welchem Falle dann das sich bildende Manganisulfat die Nitrite oxydiert.

1) Vgl. K. B. Lehmann, Die Methoden der praktischen Hygiene 2. Aufl. (1901), 228. - Rideal and Stewart, Analyst 1901, 26, 141; H. Noll, Zeitschr. f. angew. Chemie, 1905, 18, 1767; L. W. Winkler, Zeitsehr. f, analyt. Chemie 1914, 53, 665. 
Um zu exproben, ob eine geringe Menge Kaliumrhodanid die Sauerstoffbestimmung nicht beeinfluBt, wurden folgende Versuche unternommen. Es wurde ein und dieselbe Glasstöpselflasche (Vol. $236,7 \mathrm{ccm}$ ) abwechselnd einmal mit reinem luftgesättigtem destilliertem Wasser, das andere Mal mit demselben destillierten Wasser und gleichzeitig mit $0,01 \mathrm{~g}$ Kaliumrhodanid beschickt. Die Sauerstoffbestimmung wurde dann unter Verwendung von Manganosulfatlösung und verdünnter Schwefelsäure ausgeführt; die Ergebnisse waren:

Verbrauchte 1/100 N.-Natriumthiosulfatlösung:

\section{Destilliertes Wasser: \\ $27,15 \mathrm{ccm}, 27,25 \mathrm{ccm}, 27,30 \mathrm{ccm}$}

Kaliumrhodanid enthaltendes Wasser: 27,05 "27,20 " 27,20 "

Das Ergebnis ist also in beiden Fällen nahezu dasselbe. Wurde aber zum Ansäuern rauchende Salzsäure genommen, so war der Verbrauch an Thiosulfatlösung um ein Geringes niedriger, nämlich 26,70, 26,80 bezw. 26,75 cem.

Die folgenden Versuche wurden mit nitrithaltigem Wasser (im Liter $1,0 \mathrm{mg} \mathrm{N}_{2} \mathrm{O}_{3}$ ) vorgenommen, und zwar wieder in derselben Weise wie bei den ersten Versuchen, d. h. unter Benutzung einer und derselben Flasche. Das nitrithaltige Wasser wurde in der weiter unten genauer beschriebenen Weise mit Chlorkalk- und Rhodanidlösung vorbehandelt. Es möge nur noch bemerkt werden, daß zur reinen, bei Zimmerwärme mit Luft gesättigten Wasserprobe, statt der mit Glaubersalzlösung bereiteten Chlorkalk- und Rhodanidlösung dieselbe Menge $(2,5 \mathrm{ccm})$ reine Glaubersalzlösung und auch Schwefelsäure $(0,5 \mathrm{ccm})$ hinzugefügt wurde, um zu unmittelbar vergleichbaren Zahlen zu gelangen.

Verbrauchte 1/100 N.-Natriumthiosulfatlösung:

Destilliertes Wasser: $27,65 \mathrm{ccm}, 27,75 \mathrm{ccm}, 27,70 \mathrm{ccm}, 27,70 \mathrm{ccm}, 27,65 \mathrm{ccm}, 27,65 \mathrm{ccm}$ Nitrithaltig. Wasser: $27,70,27,75,27,75$ "27,80 , 27,65 " 27,60 "

Die Versuche beweisen zur Genüge, daß die sonst so störende salpetrige Säure durch die Vorbehandlung vollständig unschädlich gemacht wird.

Es wurden dann auch Versuche mit destilliertem Wasser ausgeführt, das mit einer Abkochung von Baumblättern gelblich gefärbt war, endlich auch solche, bei welchen das Wasser sowohl mit Blätterauszug als mit Nitriten (1,0 $\mathrm{mg} \mathrm{N}_{2} \mathrm{O}_{3}$ im Liter) verunreinigt war. Das Reduktionsvermögen des ersteren Wassers betrug 5,5 Grade ${ }^{1}$ ), das des letzteren 7,0 Grade.

Verbrauchte 1/100 N.-Natriumthiosulfatlösung:

Destilliertes Wasser: $26,70 \mathrm{ccm}, 26,60 \mathrm{~cm}, 26,50 \mathrm{ccm}$, Blätterabkochung enthaltendes Wasser: $26,75 \mathrm{cem}, 26,50 \mathrm{ccm}, 26,30 \mathrm{ccm}$;

Destilliertes Wasser: $26,40 \mathrm{ccm}, 26,40 \mathrm{ccm}, 26,50 \mathrm{~cm}$, Blätterabkochung und Nitrit enthaltendes Wasser: $26,20 \mathrm{ccm}, 26,30 \mathrm{ccm}, 26,20 \mathrm{ccm}$.

Die Versuche zeigen, daß das im folgenden näher beschriebene Verfahren zur Bestimmung des gelösten Sauerstoffs in nitrithaltigen und mit organischen Stoffen verunreinigten Wässern sehr geeignet erscheint.

An Lösungen werden gebraucht:

1. Manganosulfatlösung. Man löst unter gelindem Erwärmen 1 Teil kryst. Manganosulfat $\left(\mathrm{MnSO}_{4}, 4 \mathrm{H}_{2} \mathrm{O}\right)$ in 2 Teilen destillierten Wassers.

2. Kaliumjodidhaltige Natronlauge. Es werden 2 Teile reinstes Natriumhydroxyd (pro a nalysi) und 1 Teil reinstes Kaliumjodid in 4 Teilen

1) Vergl. Zeitschr. f. analyt. Chemie 1902, 41, 419. 
destilliertem Wasser gelöst. Eine kaliumjodidfreie Natronlauge ist, wenn man das Chlorkalk-Rhodanidverfahren benutzt, überflüssig.

3. Chlorkalklösung. $1 \mathrm{~g}$ guter Chlorkalk von etwa $30 \%$ wirksamem Chlorgehalt wird mit $100 \mathrm{ccm}$ starker Glaubersalzlösung $(25 \mathrm{~g}$ krystall. Natriumsulfat in destilliertem Wasser zu $100 \mathrm{ccm}$ gelöst) innigst verrieben, dann die durch Gips usw. getrübte Flüssigkeit durch einen Wattebausch filtriert. Als Lösungsmittel wỉrd eine starke Glaubersalzlösung verwendet, um eine schwere Flüssigkeit zu erbalten, die auf den Boden der mit dem Untersuchungswasser gefüllten Flaschen gegeben werden kann; andererseits kann diese Flüssigkeit praktisch als sauerstofffrei gelten.

4. Rhodanidlösung. $1 \mathrm{~g}$ reines weißes Kaliumrhodanid wird in $200 \mathrm{ccm}$ starker Glaubersalzlösung (s. o.) gelöst.

5. Verdünnte Schwefelsäure. Man mischt 3 Vol. dest. Wasser mit 2 Vol. kone. Schwefelsäure; diese annähernd $50 \%$-ige Säure wird natürlich nur nach dem Erkalten benutzt.

Die Untersuchung wird in folgender Weise vorgenommen:

a) Vorbehandlung mit Chlorkalklösung. Diese wird an der Entnahmestelle ausgefübrt. $Z u$ den in den fast ganz vollen Faschen befindlichen Wasserproben von etwa $250 \mathrm{ccm}$ wird $0,5 \mathrm{ccm}$ Chlorkalklösung und ebensoviel verdünnte $(\hat{n} 0 \%$-ige $) \mathrm{Sch}$ w e fels ä ure gegeben. Die Flaschen werden verschlossen und der Inhalt durch kräftiges Schütteln gemengt. Die mit Stöpselklammern versehenen Flaschen (Fig. 1) werden ins Laboratorium gebracht ${ }^{\mathbf{1}}$ ).

Bequemlichkeitshalber verwendet man zum rohen Abmessen der Reagenzien keine Pipetten, sondern Tropfgläser; es werden von der Chlorkalklösung und der Schwefelsäure je 10 Tropfen genommen.

Ein besonderer Vorteil der in Vorschlag gebrachten Vorbehandlung ist, dab der ursprüngliche Sauerstoffgehalt des Wasser wie Versuche zeigten - sich auch nach 24 Stunden kaum meßbar verändert. Durch Versetzen des Wassers mit der Chlorkalk.
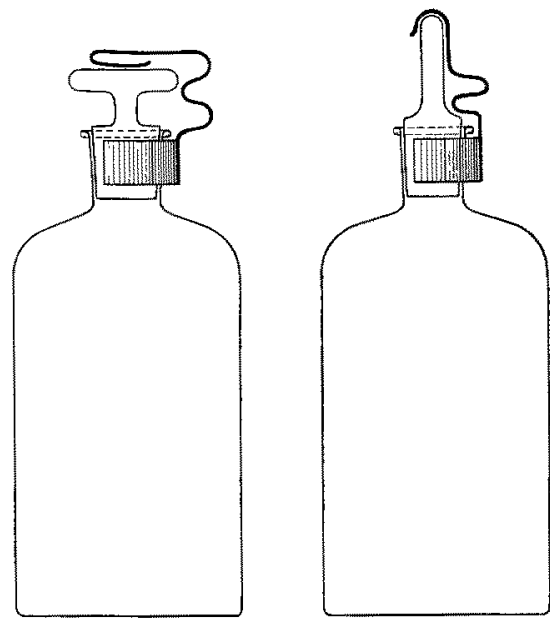

Fig. 1. lösung und Schwefelsäure werden nämlich alle Kleinwesen rasch abgetötet, auch werden die leichter oxydierbaren organischen Stoffe zerstört; die Sauerstoffzehrung des Untersuchungswassers wird also zum Stillstand gebracht.

Es möge nur noch bemerkt werden, daß, wenn man die Vorbehandlung mit Chlorkalklösung im Laboratorium vornimmt, man nach dem Hinzufügen der Chlor-

1) Die Stöpselklammern lasse man sich ans etwa $0,5 \mathrm{~mm}$ starkem hartem Messingblech machen; zweckmäßig werden die Klammern vernickelt. Verfasser zieht Flaschen mit Griffstöpsel vor.

Neuerdings werden ganz unnötigerweise auch Sauerstoff-Flaschen mit schräg abgeschliffenen Stöpseln benutzt. Ist die Flasche gehörig voll und benetzt man den Glasstöpsel vor dem Einsetzen mit Wasser, so bleibt bei einiger Aufmerksamkeit nie eine Luftblase in der Flasche. 
kalklösung und Schwefelsäure etwa 10 Minuten warten muß und dann erst die unter $\mathrm{b}$ beschriebene Vorbehandlung mit Rhodanidlösung vornehmen darf. Die Nitrite werden nämlich durch die unterchlorige Säure fast sofort oxydiert, die organischen Stoffe brauchen aber, wie schon erwähnt, in dieser stark verdünnten Lösung etwas Zeit, um unschädlich gemacht zu werden; je länger man wartet, um so genauer werden die Ergebnisse.

b) Vorbehandlung mit Rhodanidlösung. Diese wird für gewöhnlich im Laboratorium vorgenommen. Bevor nämlich die Sauerstoffbestimmung zur Ausführung gelangen kann, muß der Überschuß der vorhandenen unterchlorigen Säure

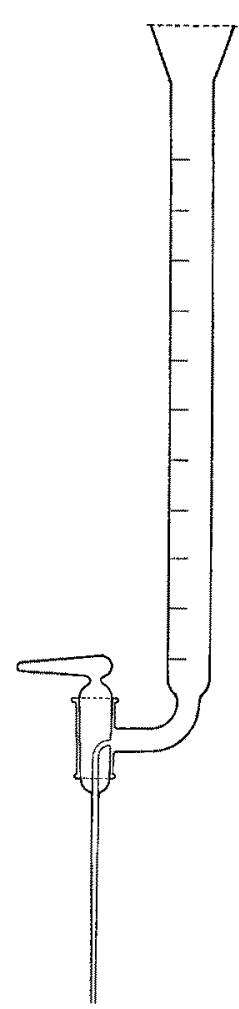

Fig. 2. reduziert werden. $\mathrm{Zu}$ diesem Zweck gibt man in die Flaschen $2 \mathrm{ccm}$ der Rhodanidlösung, verschließt wieder und mengt den Inhalt der Flaschen durch kräftiges Schwenken. Man wartet nun 10 Minuten, nach welcher Zeit schon ganz sicher nicht die geringste Menge unterchloriger Säure mehr vorhanden ist; nun wird die Sauerstoffbestimmung vorgenommen.

c) Sauerstof fbestimmung. Diese gelangt nach der unter $a$ und $b$ beschriebenen Vorbehandlung wesentlich in der alten Form zur Ausführung. Man gibt in jede Flasche $1 \mathrm{ccm}$ Manganosulfatlösung und $2 \mathrm{ccm}$ kaliumjodidhaltige Natronlauge und säuert dann mit $5 \mathrm{ccm}$ (bezw. bei sehr kaltem Wasser mit $10 \mathrm{ccm}$ ) $50 \%$-iger Schwefel säure an; bei eisenreichen Wässern nimmt man $5 \mathrm{ccm} 50 \%$-ige Phosphorsäure. Vom Flascheninhalt wird im ganzen ein $\mathrm{Abzug}$ von rund 6 ccm gemacht.

Statt die Reagenzien mit Pipetten abzumessen, kann man hierzu vortellhaft kleine in ganze Kubikzentimeter geteilte Hahnbüretten benutzen, an deren Ausflußspitze eine $10-15 \mathrm{~cm}$ lange recht enge Glasröhre angeschmolzen ist. (Fig, 2.)

Mit diesen Büretten werden dann die Reagenzien auf den Boden der Flaschen gegeben. Als Hahnfett nimmt man für die Laugenbürette eine Salbe, die aus 1 Teil Ceresin und 4 Teilen gelbem Vaselin bereitet ist.

Bezüglich der Wahl der Untersuchungsmethode sei folgendes bemerkt:

Bei der Untersuchung von destilliertem oder reinem natürlichen Wasser, ebenso bei der Untersuchung von störende Salze nicht enthaltenden wässerigenLösungen (z. B. von reinem Meerwasser) wird das ursprüngliche Verfahren (mit kaliumjodidhaltiger Natronlauge) benutzt. Es ist in diesen Fällen gleichgültig, ob man zur Bindung des Sauerstoffs Manganchlorür oder Manganosulfat und zum Ansäuern Salzsäure oder Schwefelsäure nimmt. Der einzige Unterschied ist, dab bei Verwendung von Schwefelsäure der Niederschlag etwas langsamer gelöst wird.

Sind im Wasser in reichlicher Menge organische Stoffe aber keine Nitrite enthalten, so kann man ebenfalls das ursprüngliche Verfahren (mit kaliumjodidhaltiger Natronlauge) anwenden. Um die für gewöhnlich nur mit einem kleinen Fehler behafteten Zahlen zu verbessern, wird der Verbesserungswert unter Verwendung von $1 / 100$ N.-Jodlösung bestimmt.

1) Zeitschr. f. analyt. Chemio 1914, 53, 668. 
Enthält das Wasser Nitrite, so können verschiedene Wege eingeschlagen werden. Begnügt man sich mit der annähernden Bestimmung, so benutzt man Natronlauge ohne Kaliumjodid und zum Ansäuern Salzsäure oder Schwefelsäure; das Kaliumjodid wird erst nachträglich zur Flüssigkeit gegeben. Wünscht man die so erbaltenen, zu niedrigen Zahlen zu verbessern, so kann das Verfahren mit Manganichloridlösung zur Anwendung gelangen. Die Verbesserung hilft aber nur dann wirklich, wenn im Untersuchungswasser organische Stoffe nur in geringer Menge vorhanden sind.

Zur Untersuchung von Wässern, die gleichzeitig Nitrite und in reichlicher Menge organische Stoffe enthalten, kann man entweder die „Koblensäuremethode" 1 ), (welcbe auf der Überführung des Manganohydroxydes durch Kohlensäure in Manganocarbonat beruht), benutzen, oder aber das oben beschriebene Verfahren.

Das eigentliche Anwendungsgebiet der „Kohlensäuremethode“ liegt aber mehr auf rein wissenschaftlichem Gebiete. Es können nämlich bei Benutzung dieser $\mathrm{Me}$ thode auch solche Wege betreten werden, die bisher ganz unzugänglich waren. So wurde z. B. mit diesem Verfahren der gelöste Sauerstoff in 1/100 N.- Natriumthiosulfatlösung und in $1 / 100 \mathrm{~N}$.-Natriumnitritlösung $\left(0,690 \mathrm{~g} \mathrm{NaNO}_{2}\right.$ im Liter) bestimmt; in $1000 \mathrm{ccm}$ mit Luft gesättigter Lösung wurden bei $20^{\circ}$ (Barometerstand $760 \mathrm{~mm}) 6,13$ bezw. $6,31 \mathrm{ccm}$ Sauerstoff gefunden.

Bei praktischen Wasseruntersuchungen dürfte sich aber das jetzt veröffentlichte Verfahren behaupten, nicht nur seiner Einfachheit halber, sondern besonders deshalb, weil in verunreinigten Wässern kolloidal gelöste Stoffe vorhanden sind, die bei der „Kohlensäuremethode“ vom Niedersehlag mitgerissen werden können und dementsprechend das Ergebnis erniedrigen, während beim Chlorkalk-Rhodanidverfahren die störenden Stoffe schon bei der Vorbehandlung unschädlich gemacht werden.

$\mathrm{Um}$ nicht an verschiedene Untersuchungsmethoden gebunden zu sein, dürfte es in der Praxis das einfachste sein, ob nun ein verunreinigtes oder ein reines Wasser vorliegt, die Vorbehandlung mit Chlorkalk- und Rhodanidlösung, schon der einheitlichen Arbeitsweise zuliebe, in allen Fällen durchzuführen. Bei verunreinigten Wässern wird erst hierdurch die Bestimmung ermöglicht, und bei reinen Wässern hat man keinen Nachteil.

Es möge nur nochmals hervorgehoben werden, daß man nach dem Hinzufügen der Rhodanidlösung gut mischen und einige Zeit (etwa 10 Minuten) warten muß, um ja nicht etwa wirksames Chlor anstatt Sauerstoff zu messen. Bevor man sich auf die Methode eingeübt hat, wird man gut tun, nach Beendigung der Vorbehandlung sich von der Abwesenheit von wirksamem Chlor zu überzeugen, indem man in die Flasche etwas $10 \%$-ige mit Stärkelösung bereitete Kaliumjodidlösung träufelt. $\mathrm{Zu}$ dieser Probe kann aber gewöhnliches käufliches Kaliumjodid nicht verwendet werden, welches fast immer infolge eines Jodatgehaltes einen geringen Gehalt an wirksamem Chlor (auch in mit Schwefelsäure angesäuertem dest. Wasser) vortäuscht, sondern man darf nur reinstes Kaliumjodid (pro analysi) benutzen.

Bezüglich der Genauigkeit des in dieser Abhandlung beschriebenen Verfahrens bemerke ich folgendes: Bis zu einigen Graden Reduktionsvermögen sind die Ergebnisse fast so genau wiebei reinem Wasser; es istgleich. gültig, ob das Wasser Nitrite enthält oder nicht. Bei Wässern mit bedeutendem Reduktionsvermögen wird man aber schon etwas weniger Sauerstoff finden, 
als wirklich vorhanden ist. Man kann bei einer Zunabme des Reduktionsvermögens um je $10^{\circ}$ auf einen Fehler in der Sauerstoffbestimmung von etwa - 0,8\% rechnen. So wurden z, B. in einem nitrithaltigen verunreinigten Wasser, welches ein Reduktionsvermögen von $63^{\circ}$ besaß, im Liter anstatt 6,42 nur 6,09 ccm Sauerstoff gefunden; der Wert ist also auch bei diesem ausnahmsweise stark verunreinigten Wasser nur um etwa 5\% zu klein.

Bei natürlichen Wässern oder Abwässern, die mit organischen Stoffen beladen sind, werden aber alle bisher erwäbnten Verfahren versagen. Solche Wässer

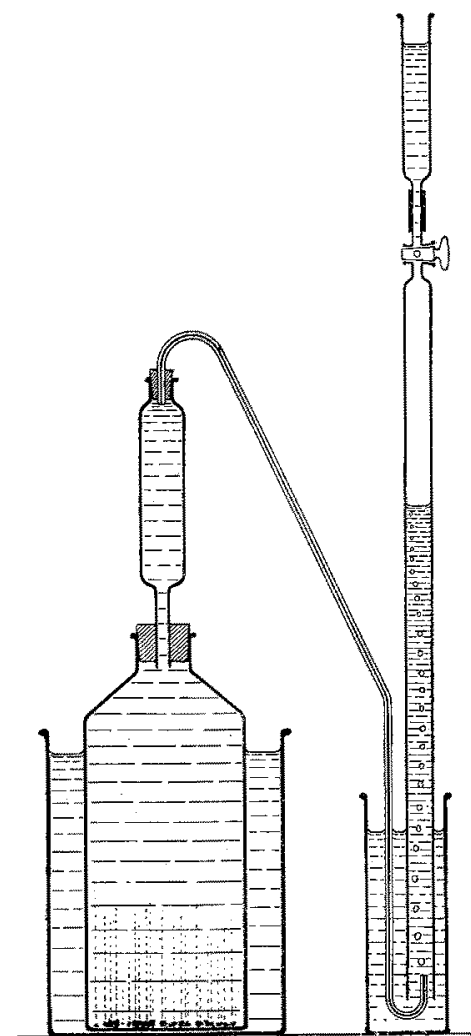

Fig. 3. sind zwar meist im frischen Zustande so gut wie sauerstofffrei; soll aber dennoch die Menge des allenfalls gelösten Sauerstoffs bestimmt werden, so kanı dies nur auf gasvolumetrischem Wege erfolgen.

Zur Bestimmung der in natürlichen Wässern gelösten Gase hat Verf. schon vor Jahren ein einfaches gasanalytisches Verfahren angegeben ${ }^{1}$ ), welches auch an Ort und Stelle ausgefübrt werden kann, wobei man den Luftdruck mit einem geprüften Aneroidbarometer miBt. Die Ausführungsform dieses Verfahrens wurde jetzt verbessert und soll kurz beschrieben werden.

In eine ausgemessene Glasstöpselflasche von etwa $500 \mathrm{ccm}$ streut man $10 \mathrm{~g}$ grobkörnigen von feinem Staube durch Sieben gereinigten weißen Ma rmor; früher wurde Calcit vorgeschrieben, wie aber neuere Versuche zeigten, kann man auch Marmor nehmen. Auf den Marmor wird wenig mit Salzsäure angesäuertes Wasser gegossen; nach $1-2$ Minuten währender Gasentwickelung wird die Flüssigkeit abgegossen; der Zweck dieses Vorgehens ist, den Marmor gleichmäßig zu befeuchten. Das zu untersuchende $W$ asser wird nun längere Zeit auf geeignete Weise durch die Flasche geleitet, bis es sich in der Flasche sicher erneuert hat. Wird die Bestimmung des gelösten Sauerstoffs nicht an der Entuahmestelle ausgeführt, so gibt man auf den Boden der Flasche, um die Sauerstoffzehrung zu hemmen, $1-2 \mathrm{ccm}$ von der glaubersalzhaltigen Chlorkalklösung (s. Seite 123); der Inhalt der verschlossenen Flasche wird gemischt. Die mit einer dünnen Gummikappe verbundene Flasche wird im Laboratorium geöffnet und der aus der Zeichnung (Fig. 3) ersichtliche $20 \mathrm{cem}$ fassende A ufsatz eingesetzt; früher wurde kein Aufsatz verwendet. Man drückt den Gummistöpsel so tief in den Flaschenhals, daß das Wasser in der Mitte der etwa $8 \mathrm{~mm}$ weiten Röhre des Aufsatzes steht; sollte es in der Flasche an Wasser mangeln, so fügt man erst $2-3 \mathrm{~cm}$ gesättigte (luftfreie) Chlorcalciumlösung hinzu. Man stellt die Flasche in ein großes starkes Becherglas (oder in ein BlechgefäßB) und füllt den Aufsatz bis zum Halse mit rauchender Salzsäure (spez. Gew. 1,18); auf die Salzsäure wird 1-2 cem Wasser geschichtet. Die Gasentwickelung beginnt erst nach einigen

1) Zeitschr. f. analyt. Chemie 1901, 40, 523. 
Minuten, sodaß Zeit bleibt, den schon vorbereiteten, mit einer dickwandigen sehr engen Gasleitungsröhre versehenen kleinen Gummistöpsel in den Hals des ganz vollen Aufsatzes einzupassen, sowie auch die mit $20 \%$-iger Natronlauge gefüllte $30-40 \mathrm{~cm}$ lange und etwa $1 \mathrm{~cm}$ weite Meßröhre auf das Ende des Gasleitungsrohres zu stellen. Beim Einsetzen des Gasleitungsrohres achte man darauf, daß es sich mit Wasser ganz füllt, also keine Luftblasen in ihm zurückbleiben. Das in sehr kleinen Bläschen sich entwickelnde Kohlendioxyd reißt die in der Flüssigkeit gelösten Gase mit und überführt sie auf diese Weise in die Meßröhre.

Ist das Untersuchungswasser eiskalt, so ist die Gasentwickelung zu träge, ist es warm (über $20^{\circ}$ ), so ist die Entwickelung zu stürmisch. Deshalb gießt man in das große Becherglas je nach der Temperatur des Untersuchungswassers warmes oder kaltes Wasser, um die Geschwindigkeit der Gasentwickelung zu regeln. Die Menge der rauchenden Salzsäure $(20 \mathrm{ccm})$ ist so bemessen, daß die Gasentwickelung am regelmäßigsten bei $15^{\circ}$ erfolgt. Man kann auch im Winter einen etwas größeren, im Sommer einen kleineren Aufsatz verwenden.

Um die vollständige Bindung des Kohlendioxyds durch die Natronlauge sicher zu stellen, läßt man von Zeit zu Zeit in die Meßröhre aus der daran befindlichen Trichterröhre etwas frische Lauge zufließen. Nach etwa 30 Minuten ist der Versuch beendet. - Bezüglich des Messens des Gases verweise ich auf die schon erwähnte Abhandlung.

Bei den früheren Versuchen wurde zum Binden des Sauerstoffgases alkalische Pyrogallollösung benutzt; man kann aber hierzu auch Natriumhydrosulfitlösung verwenden (Franzen). Man gibt in ein Glasfläschchen von $10 \mathrm{ccm}$ Inhalt $2 \mathrm{~g}$ hydroschwefligsaures Natrium $\left(\mathrm{Na}_{2} \mathrm{~S}_{2} \mathrm{O}_{4}\right)$, füllt das Fläschchen mit N.-Natronlauge, verschließt mit einem mit Paraffin getränkten Korke und schüttelt sofort bis zum Lösen des Salzes, Diese Flüssigkeit wird dann in die Trichterröhre gegeben. Mit dieser Lösung arbeitet man reinlicher als mit der sich bräunenden alkalischen Pyrogallollösung, jedoch geht die Bindung des Sauerstoffs langsamer vonstatten.

Bei der Berechnung kommt vom Inhalte der Flasche das Volumen des Marmors $(3,6 \mathrm{ccm})$ und die allenfalls hinzugefügte glaubersalzhaltige Chlorkalklösung in Abzug. In den $20 \mathrm{ccm}$ luftgesättigter rauchender Salzsäure (von $38 \%$ ) sind nach meiner Bestimmung 0,192 ccm Stickstoff- und 0,083 ccm Sauerstoffgas gelöst, welche von der in der Meßröhre befindlichen Gasmenge abgezogen werden.

Endlich möchte ich noch kurz bezüglich meiner Untersuchungen über die Löslichkeit der atmosphärischen Luft in Wasser bemerken, daß ich über diesen Gegenstand zuerst im Jahre 1888 schrieb, indem ich auf Grund gasanalytischer Bestimmungen nachwies, daß der Sauerstoffgehalt der bei verschiedenen Temperaturen im Wasser gelösten Luft nicht stets derselbe bleibt (zwischen $1^{0}$ und $23^{\circ}$ ) wie Bunsen ${ }^{1}$ ) gefunden hatte, sondern mit der Zunahme der Temperatur fortwährend abnimmt ${ }^{2}$ ). Ich bestimmte ${ }^{3}$ ) die Absorptionskoeffizienten des Sauerstoff- und Stickstoffgases im Absorptiometer, also auf physikalischem Wege, und berechnete die Löslichkeit der Luft in Wasser auf Grund des Gesetzes von Henry-Dalton ${ }^{4}$ ). Auch entfernte ich aus luftgesättigtem Wasser die gelöste Luft wieder durch Auskochen und bestimmte den Sauerstoffgehalt des Gasgemenges mit größtmöglicher

1) Gasometrische Methoden 2. Aufl., 224.

2) Ber. D. Chem. Ges. 1888, 21, 2851.

3) Ber. D. Chem. Ges. 1891, 24, 3602.

$\left.{ }^{4}\right)$ Ber. D. Chem. Ges. 1901, 34, 1408. 
Genauigkeit in einem besonderen Eudiometer durch Verbrennen mit überschüssigem Wasserstoff. Aus dem so ermittelten Sauerstoffgehalt der ausgekochten Luft ergibt sich folgende Formel, die den Sauerstoffgehalt $(n)$ der im Wasser gelösten Luft zwischen $0-30^{\circ}$ in Prozenten anzeigt:

$$
n=34,91-0,0438 \mathrm{t} \text {. }
$$

Der Fehler im Sauerstoffgehalte dürfte bei den mit dieser Formel berechneten Zahlen kaum $0,01 \%$ betragen.

Da ich die Löslichkeit des Luftsauerstoffs schon früher jodometrisch genau bestimmt hatte ${ }^{1}$ ), konnte der in luftgesättigtem Wasser enthaltene Stickstoff (mit seinen Begleitern) unabhängig davon, ob die Bestandteile der Luft beim Lösen in Wasser dem Gesetze von Henry-Dalton genau folgen oder nicht, mit voller Genauigkeit berechnet werden. Die auf diesem Wege erhaltenen Zahlen sind dann in Lunge's Handbuch der Chemisch-technischen Untersuchungsmethoden 1904, Bd. I, 322, veröffentlicht worden.

1) Ber. D. Chem. Ges. 1889, 22, 1764.

\section{Referate.}

\section{Mehle und Backwaren.}

0. Klein: Uber portugiesische Weizensorten und ihreVeredelung. (Landw. Jahrb. 1912, 42, 329-364.) - Mit der Weizenkultur ist man in Portugal noch weit zurück. Noch vor 15 Jahren pflegte man dort mit 6-7-jährigen Brachen zu arbeiten und als einzige Düngung vor der Bestellung das während der langen Brachzeit aufgeschossene Buschwerk zu verbrennen. Seit der rationellen Verwendung von Kunstdünger konnten zwar alljährlich Ernten erzielt werden, doch genügte die Weizenproduktion auch dann bei weitem noch nicht, um den einheimischen Bedarf zu decken. 1900 mußten noch $136000 \mathrm{t}$ Weizen eingefübrt werden. Auf rationell bearbeitetem Boden kann man in Portugal bei sehr günstigen Witterungsverhältnissen die 20-25-fache Menge Korn ernten. Um die portugiesischen Weizensorten ertragsfähiger zu machen, wurden Kreuzungsversuche angestellt. Von den erzielten Sorten hat besonders eine aus dem Jahre 1902, Hybrido Belem genannt, inzwischen eine weite Verbreitung im Lande gefunden. Sie ist ausgezeichnet durch hohe Ertragsfähigkeit, Widerstandsfähigkeit gegen parasitäre Krankheiten, Ausgiebigkeit des Mehles und angenehmen Geschmack des daraus hergestellten Brotes. Die chemische Zusammenstellung dieser Weizenart ist die folgende:

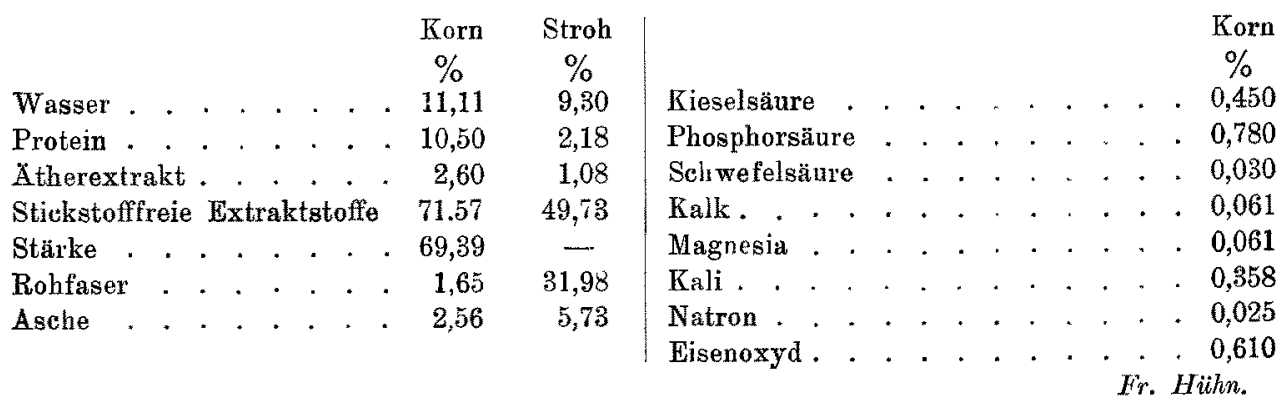

